\title{
Pluriactivité et mode de financement des exploitations agricoles
}

off-farm work status and the financial structure of the farm

\section{Stéphane Krebs}

\section{(2) OpenEdition}

1 Journals

\section{Édition électronique}

URL : http://journals.openedition.org/economierurale/148

DOI : 10.4000/economierurale. 148

ISSN : 2105-2581

\section{Éditeur}

Société Française d'Économie Rurale (SFER)

\section{Édition imprimée}

Date de publication : 30 décembre 2005

Pagination : 91-105

ISSN : 0013-0559

\section{Référence électronique}

Stéphane Krebs, "Pluriactivité et mode de financement des exploitations agricoles », Économie rurale [En ligne], 289-290 | Septembre-décembre 2005, mis en ligne le 03 mars 2009, consulté le 19 avril 2019. URL : http://journals.openedition.org/economierurale/148 ; DOI : 10.4000/economierurale.148

Ce document a été généré automatiquement le 19 avril 2019.

(c) Tous droits réservés 


\title{
Pluriactivité et mode de financement des exploitations agricoles
}

Off-farm work status and the financial structure of the farm

\author{
Stéphane Krebs
}

\section{NOTE DE L'ÉDITEUR}

Lors de la finalisation de cet article (premier semestre 2005) l'auteur, en thèse de doctorat, appartenait au laboratoire d'Économie forestière de Nancy.

1 L'agriculture française a connu, depuis la fin de la Seconde Guerre mondiale d'importantes mutations. Le fait le plus notable réside probablement dans l'affirmation du caractère entrepreneurial de l'activité de production agricole et l'émergence d'un modèle universel d'exploitation agricole, articulée autour de deux actifs familiaux et dégageant un revenu suffisant pour garantir à l'agriculteur et à sa famille un niveau de vie satisfaisant. Cette revalorisation du statut social de l'agriculteur est à l'origine d'un formidable mouvement de modernisation des exploitations, matérialisé par un développement de l'investissement productif et une généralisation du recours au crédit bancaire. Ces bouleversements, ardemment souhaités par la profession et accompagnés par les pouvoirs publics dans le cadre des politiques agricoles nationales et communautaires, ont conduit à reléguer à l'arrière-plan la pluriactivité des familles agricoles, qui constitue pourtant un trait permanent des sociétés rurales.

Ces choix politiques, s'ils ont contribué à remodeler en profondeur la physionomie du secteur et permis un formidable accroissement du potentiel productif agricole français, n'ont pas entrâné une disparition de la pluriactivité des familles d'agriculteurs, bien au contraire. Au cours de la période récente, cette dernière a même gagné l'agriculture professionnelle, abandonnant progressivement le champ de l'agriculture de complément où elle était originellement cantonnée. Le visage de la pluriactivité s'est également modifié, puisque les situations de double activité des chefs d'exploitation ont eu tendance 
à régresser, alors même que l'exercice d'une activité extérieure par le conjoint de l'exploitant progressait, du fait notamment du développement de l'activité féminine. Ce développement de la pluriactivité dans le champ de l'agriculture professionnelle coïncide avec les changements récents intervenus dans les modalités de soutien à l'agriculture et les difficultés rencontrées par certaines catégories de producteurs, qui tendent à fragiliser l'équilibre économique et financier de leurs exploitations. Les agriculteurs ont alors pu être légitimement incités, pour restaurer leur position financière, à opérer une diversification de leurs sources de revenus et d'activités, encouragée par ailleurs par les pouvoirs publics depuis la réforme de la Politique agricole commune (PAC) de 1992 au titre des politiques de développement rural et d'aménagement du territoire.

3 La question des relations que sont susceptibles d'entretenir les décisions de pluriactivité des familles d'agriculteurs et le financement de leur exploitation, qui revêt une importance particulière dans le contexte actuel, a été maintes fois évoquée, sans avoir toutefois pu faire l'objet, jusqu'à présent, d'investigations approfondies. La pluriactivité a en effet donné lieu à une abondante littérature économique (Lass, Findeis et Hallberg, 1991 ; OCDE, 2001'1), mais celle-ci a essentiellement mis l'accent, au plan théorique comme au plan empirique, sur l'identification des déterminants de la décision de participation à une activité extérieure, en insistant sur l'influence des caractéristiques individuelles et familiales (âge, formation agricole et générale, expérience professionnelle, taille et composition de la famille, etc.), ainsi que de celles de l'exploitation (dimension, orientation technico-économique, etc.) et de sa localisation. La dimension financière n'est pas totalement absente dans ces travaux, mais son influence réelle sur les décisions de travail des familles d'agriculteurs tend à demeurer largement sous-estimée. Les revenus d'activité extérieure pourraient en effet contribuer au financement des investissements sur l'exploitation, ce qui serait de nature à rendre l'activité agricole plus compétitive et pourrait expliquer la persistance de l'agriculture à temps partiel dans les pays développés (Schmitt, 1991). Mais la recherche d'un complément de ressources hors de l'exploitation pourrait également renvoyer plus simplement, comme le suggèrent Brangeon, Jégouzo ou Roze (1994), à une logique de compensation d'un mauvais résultat agricole.

4 La présente étude ${ }^{2}$ entend ainsi contribuer au renouvellement des approches économiques de la pluriactivité agricole, en privilégiant une analyse dynamique des divers comportements des familles d'agriculteurs. Ce travail, original et inédit, a été rendu réalisable par la possibilité de construire un panel d'exploitations agricoles françaises. Ce panel autorise une mise en relation du statut des exploitations en matière de pluriactivité avec les principales caractéristiques structurelles et économiques des exploitations, ainsi qu'avec leurs caractéristiques financières. Ces données permettent de faire ressortir certaines spécificités des exploitations concernées par le phénomène, tant en matière de financement interne (autofinancement) que de financement externe (endettement).

\section{Une source statistique inédite}

5 Les données employées dans cet article, qui autorisent une analyse longitudinale des décisions d'emploi, d'investissement et de financement des familles d'agriculteurs, ont pour origine deux enquêtes, réalisées par l'Institut national de la statistique et des études économiques (INSEE) et mettant en relation, pour les exercices comptables 1991 et 1997, les données du Réseau d'information comptable agricole (RICA) et des déclarations 
fiscales d'agriculteurs présents dans cette source. Le caractère historique du RICA permet la construction d'un échantillon permanent qui, mis en relation avec les données de ces enquêtes, a donné naissance au panel (cylindré) RICA-Source fiscale, composé de 1712 exploitations individuelles ${ }^{3}$, appartenant au champ de l'agriculture professionnelle et observées sur la période 1991-1997.

6 La principale difficulté réside dans l'identification des exploitations concernées par la pluriactivité, puisque la source comptable ne contient aucune indication quant au statut de l'exploitant et/ou de sa famille en matière d'emploi. Les données fiscales s'avèrent alors particulièrement précieuses, dans la mesure où elles permettent de disposer, de manière détournée, d'éléments concernant le statut en matière de pluriactivité. Il est en effet possible de construire une typologie des foyers d'agriculteurs, en définissant pour l'exploitant et son conjoint une variable "revenu d'activité non agricole », par agrégation des traitements et salaires et des bénéfices non agricoles (bénéfices industriels et commerciaux, bénéfices non commerciaux) déclarés à l'administration fiscale. La pluriactivité peut alors être définie à l'échelon individuel, en considérant comme pluriactif tout individu dont le montant du « revenu d'activité non agricole » excède un seuil de revenu, jugé significatif et correspondant au quart du salaire annuel moyen publié par l'INSEE pour l'année considérée. La définition de la pluriactivité peut ensuite être étendue au foyer de l'agriculteur, en considérant comme pluriactif tout foyer dont un membre du couple au moins est considéré individuellement comme pluriactif.

7 Il en résulte une source statistique singulière, permettant de disposer de l'intégralité des informations structurelles, économiques et financières du RICA sur l'ensemble de la période étudiée (1991-1997), ainsi que d'une indication quant au statut en matière d'emploi en début et en fin de période d'observation (1991 et 1997).

8 La prise en compte de la dimension individuelle, rendue possible par l'utilisation de données de panel, laisse entrevoir un développement timide de la pluriactivité sur les exploitations individuelles appartenant au champ de l'agriculture professionnelle, puisque la part des exploitations concernées par le phénomène dans l'échantillon ne progresse que de $21 \%$ environ en 1991 à un peu plus de $22 \%$ en 1997 (tableau1). Cette progression contraste sensiblement avec celle observée au cours de la dernière décennie à partir des données du Recensement général de l'agriculture, puisque la part des exploitations pluriactives progresse dans l'agriculture de $27 \%$ à $36 \%$ entre 1988 et 2000 . Dans la seule agriculture professionnelle, qui ne concerne que $60 \%$ des exploitations mais qui contribue pour plus de $95 \%$ à la production agricole française, le développement de la pluriactivité est même spectaculaire, puisqu'elle ne concernait qu'une exploitation sur cinq environ en 1988 contre près d'une exploitation sur trois à l'heure actuelle ${ }^{4}$.

Une analyse plus fine, tenant compte des changements de statut des foyers d'agriculteurs en matière d'emploi intervenus entre 1991 et 1997 et permettant d'opposer quatre catégories de foyers ("foyers monoactifs en 1991 et 1997 ", "foyers pluriactifs en 1991 uniquement », " foyers pluriactifs en 1997 uniquement » et " foyers pluriactifs en 1991 et 1997 ») laisse entrevoir une inertie importante des comportements d'emploi, puisque près de $90 \%$ des exploitations ne sont pas concernés par un changement de statut en matière d'emploi au cours de la période étudiée ${ }^{5}$. 
Tableau 1. Répartition des foyers selon leur statut en matière de pluriactivité en 1991 et 1997

\begin{tabular}{|l|l|l|l|l|}
\hline & & Statut & en & 1997 \\
\hline & & Foyers monoactifs & Foyers pluriactifs & Ensemble \\
\hline Statut & Foyers monoactifs & $\begin{array}{l}1257 \\
(73,4 \%)\end{array}$ & $\begin{array}{l}102 \\
(6,0 \%)\end{array}$ & $\begin{array}{l}1359 \\
(79,4 \%)\end{array}$ \\
\hline en & Foyers pluriactifs & $\begin{array}{l}75 \\
(4,4 \%)\end{array}$ & $\begin{array}{l}278 \\
(16,2 \%)\end{array}$ & $\begin{array}{l}353 \\
(20,6 \%)\end{array}$ \\
\hline 1991 & Ensemble & $\begin{array}{l}1352 \\
(77,8 \%)\end{array}$ & $\begin{array}{l}\mathbf{3 8 0} \\
(22,2 \%)\end{array}$ & $\begin{array}{l}1712 \\
(100,0 \%)\end{array}$ \\
\hline
\end{tabular}

Source : Panel RICA-Source fiscale 1991-1997 (résultats non extrapolés)

\section{La pertinence des déterminants traditionnels de la pluriactivité}

L'examen de quelques caractéristiques structurelles de l'exploitation (tableau2) permet de faire apparaitre que la pluriactivité n'est pas systématiquement l'apanage des unités de production de faible dimension, fragilisant quelque peu l'opinion communément admise selon laquelle les foyers d'agriculteurs s'engageraient plus volontiers en pluriactivité du fait d'une faiblesse chronique de leur revenu agricole, induite par une dimension insuffisante de l'exploitation. La mise en relation du statut en matière d'emploi et de la dimension de l'exploitation produit en effet des résultats sensibles à l'indicateur de dimension retenu. L'adoption d'un critère de dimension physique comme la superficie agricole utile (SAU) laisse entrevoir une dimension a priori supérieure des exploitations concernées par la pluriactivité, tandis que l'adoption d'un critère de dimension économique comme la marge brute standard (MBS) laisserait plutôt à penser que ce sont les exploitations non concernées par la pluriactivité qui bénéficieraient d'une dimension supérieure, même si l'ampleur des différences constatées demeure dans l'ensemble relativement limitée. D'autres critères, comme l'importance de la main d'œuvre familiale ou totale employée jouent plus nettement en défaveur des exploitations durablement concernées par la pluriactivité. La prise en compte des changements de statuts intervenus en matière d'emploi au cours de la période étudiée tend ainsi à confirmer la dimension inférieure des « exploitations pluriactives en 1991 et 1997 », mais fait au contraire apparaître une dimension assez nettement supérieure - quel que soit l'indicateur de dimension retenu - pour les exploitations dont le foyer n'est que temporairement concerné par le phénomène, la dimension moyenne de ces deux dernières catégories d'exploitations excédant même celle des « exploitations monoactives en 1991 et $1997 »$.

11 Le statut en matière d'emploi semble plus volontiers corrélé à certaines autres caractéristiques économiques et structurelles de l'exploitation. 
12 Les exploitations durablement concernées par la pluriactivité sont en effet plus enclines à mettre en œuvre des techniques de production extensives - comme en témoigne le produit brut par hectare plus faible observé pour les « foyers pluriactifs en 1991 et 1997 » - et se recrutent plutôt parmi les exploitations dont la spécialisation s'accompagne de besoins en terres importants et caractérisées par une relative souplesse en matière d'emploi de la main d'œuvre (Butault et al., 1999 ; Krebs, 2004).

Tableau 2. Principales caractéristiques structurelles des exploitations agricoles

\begin{tabular}{|c|c|c|c|c|c|}
\hline & Exploitations & Exploitations & Exploitations & Exploitations & Ensemble \\
\hline & monoactives & monoactives & pluriactives & pluriactives & des \\
\hline & en 1991 et & en 1991 & en 1997 & en 1991 et & exploitations \\
\hline & 1997 & uniquement & uniquement & 1997 & \\
\hline $\begin{array}{l}\text { SAU totale (en } \\
\text { ha) }\end{array}$ & 55,9 & 56,5 & 62,2 & 63,3 & 57,5 \\
\hline $\begin{array}{l}\text { Marge brute } \\
\text { standard } \\
\text { (en milliers } \\
\text { d'UCE) }\end{array}$ & 56,5 & 58,9 & 69,8 & 55,3 & 57,2 \\
\hline $\begin{array}{lr}\text { Nombre } & \text { total } \\
\text { d'actifs } & \text { (en } \\
\text { UTA) } & \end{array}$ & 1,73 & 1,63 & 1,73 & 1,43 & 1,68 \\
\hline $\begin{array}{lr}\text { dont } & \text { actifs } \\
\text { familiaux } & \text { (en } \\
\text { UTA) } & \end{array}$ & 1,42 & 1,19 & 1,28 & 1,13 & 1,36 \\
\hline $\begin{array}{l}\text { Age du chef } \\
\text { d'exploitation }\end{array}$ & 45,4 & 42,0 & 41,6 & 42,7 & 44,6 \\
\hline $\begin{array}{l}\text { Produit brut } \\
\text { (en milliers } € \\
\text { constants 1997) }\end{array}$ & 112,2 & 113,8 & 126,0 & 103,2 & 111,6 \\
\hline $\begin{array}{l}\text { Produit brut } \\
\text { par ha }\end{array}$ & 2,0 & 2,0 & 2,0 & 1,6 & 1,9 \\
\hline $\begin{array}{l}\text { Revenu } \\
\text { agricole brut } \\
\text { (en milliers } € \\
\text { constants 1997) }\end{array}$ & 35,5 & 35,9 & 37,5 & 31,0 & 34,9 \\
\hline
\end{tabular}

Source : Panel RICA-Source fiscale 1991-1997 (résultats non extrapolés). 


\section{Pluriactivité et situation financière de l'exploitation}

13 font également apparaître un dynamisme important des exploitations concernées par la pluriactivité (tableau 3). Ces dernières sont en effet caractérisées par des besoins globaux de financement supérieurs, qui sont la conséquence d'investissements importants, mais également d'un endettement élevé, à l'origine de remboursements d'emprunts eux aussi conséquents. De plus, la perception de revenus d'activité extérieure semble agir sur les conditions d'accès au financement interne, comme le suggère le montant supérieur de l'autofinancement brut, y compris pour les exploitations durablement concernées par la pluriactivité qui ne disposent pourtant que d'une capacité réelle d'autofinancement limitée. Ce résultat remarquable est le fruit de comportements de prélèvements singuliers, les exploitations concernées par la pluriactivité ayant la possibilité de financer une large part de leurs besoins familiaux directement à partir des revenus d'origine non agricole qu'ils perçoivent. Ces différents faits stylisés confortent notre intuition initiale selon laquelle les préoccupations financières ne sont pas totalement absentes dans les décisions de pluriactivité, mais la principale difficulté réside toutefois dans l'interprétation de ces résultats observés, qui peuvent faire l'objet de deux lectures antagonistes, liées au fait de savoir s'ils constituent une cause de l'entrée en pluriactivité ou s'ils en sont, au contraire, la conséquence.

Cette question a pu être partiellement tranchée en mettant en relation la fréquence de la pluriactivité et celle des difficultés financières auxquelles ont été confrontées les exploitations au cours de la période étudiée (tableau4) ${ }^{6}$. Sur l'ensemble de la période considérée, il ne nous a ainsi pas été possible de mettre en évidence de différences marquées pour les exploitations n'ayant pas connu de changement de statut en matière d'emploi entre 1991 et 1997, si ce n'est peut-être une fragilité financière un peu plus accentuée pour les exploitations durablement concernées par la pluriactivité. Les exploitations dont le foyer n'est que temporairement concerné par le phénomène semblent par contre avoir connu une situation financière plus dégradée au cours de la période considérée (moindre représentation des exploitations présentant un risque financier nul, propension à une plus forte représentation des exploitations présentant un risque faible, moyen ou élevé).

Tableau 3. Caractéristiques financières des exploitations agricoles (moyenne, en milliers d'euros constants 1997)

\begin{tabular}{|l|c|c|c|c|c|}
\hline & Exploitations & Exploitations & Exploitations & Exploitations & Ensemble \\
\hline & monoactives & pluriactives & pluriactives & pluriactives & des \\
\hline & en 1991 et & en 1991 & en 1997 & en 1991 et & exploitations \\
\hline $\begin{array}{l}\text { Éléments du } \\
\text { tableau de } \\
\text { financement }\end{array}$ & 1997 & uniquement & uniquement & 1997 & \\
\hline
\end{tabular}




\begin{tabular}{|c|c|c|c|c|c|}
\hline 1. -EMPLOIS & 27,6 & 33,4 & 38,4 & 30,0 & 28,9 \\
\hline $\begin{array}{l}\text { - Investissement } \\
\text { total }\end{array}$ & 13,3 & 18,4 & 19,5 & 15,2 & 14,2 \\
\hline $\begin{array}{l}\text { - Remboursement de } \\
\text { dettes financières }\end{array}$ & 12,5 & 13,2 & 16,8 & 13,2 & 12,9 \\
\hline $\begin{array}{l}\text { - Variation du } \\
\text { besoin en fonds de } \\
\text { roulement }\end{array}$ & 1,7 & 1,7 & 1,9 & 1,6 & 1,7 \\
\hline - Charges à répartir & 0,0 & 0,0 & 0,0 & 0,0 & 0,0 \\
\hline $\begin{array}{l}\text { - Variation des } \\
\text { disponibilités }\end{array}$ & 0,0 & 0,1 & 0,2 & 0,0 & 0,0 \\
\hline $\begin{array}{l}\text { 2. FINANCEMENT } \\
\text { DES EMPLOIS }\end{array}$ & 27,6 & 33,4 & 38,4 & 30,0 & 28,9 \\
\hline A.Autofinancement & 15,2 & 17,9 & 20,6 & 16,2 & 15,8 \\
\hline $\begin{array}{l}\text { - Capacité } \\
\text { d'autofinancement }\end{array}$ & 35,8 & 36,2 & 37,7 & 31,4 & 35,2 \\
\hline $\begin{array}{l}\text { - Prélèvements } \\
\text { privés nets }\end{array}$ & 20,6 & 18,3 & 17,1 & 15,2 & 19,4 \\
\hline $\begin{array}{l}\text { B .Subventions } \\
\text { d'équipement } \\
\text { reçues }\end{array}$ & 0,4 & 1,3 & 0,6 & 0,6 & 0,5 \\
\hline $\begin{array}{l}\text { C.Besoin de } \\
\text { financement } \\
\text { externe }\end{array}$ & 11,9 & 14,2 & 17,2 & 13,2 & 12,5 \\
\hline $\begin{array}{l}\text { - Nouvelles dettes } \\
\text { financières }\end{array}$ & 11,8 & 14,0 & 16,9 & 13,0 & 12,4 \\
\hline $\begin{array}{l}\text { - Variation concours } \\
\text { bancaires et } \\
\text { découverts }\end{array}$ & 0,2 & 0,3 & 0,3 & 0,2 & 0,2 \\
\hline $\begin{array}{l}\text { Endettement total } \\
\text { (bilan) }\end{array}$ & 77,5 & 93,9 & 100,1 & 75,6 & 79,3 \\
\hline $\begin{array}{l}\text { - Dettes financières } \\
\text { à LMT* }\end{array}$ & 51,5 & 64,9 & 66,4 & 49,4 & 52,7 \\
\hline $\begin{array}{l}\text { - Dettes financières } \\
\text { à } \mathrm{CT}^{* *}\end{array}$ & 4,0 & 3,3 & 4,0 & 4,2 & 4,0 \\
\hline
\end{tabular}


* Long et moyen terme. ** Court terme.

Source : Panel RICA-Source fiscale 1991-1997 (résultats non extrapolés).

Tableau 4. Typologie du risque financier (moyenne 1991-1997)

\begin{tabular}{|l|l|l|l|l|}
\hline & Exploitations & Exploitations & Exploitations & Exploitations \\
\hline & monoactives & pluriactives & pluriactives & pluriactives \\
\hline & en 1991 et & en 1991 & en 1997 & en 1991 et \\
\hline & 1997 & uniquement & uniquement & 1997 \\
\hline Risque financier : & & & & \\
\hline -Nul & $53,3 \%$ & $46,9 \%$ & $42,6 \%$ & $50,9 \%$ \\
\hline -Faible & $23,1 \%$ & $27,2 \%$ & $32,9 \%$ & $23,2 \%$ \\
\hline -Moyen & $18,4 \%$ & $20,4 \%$ & $18,1 \%$ & $18,4 \%$ \\
\hline -Elevé & $5,3 \%$ & $5,5 \%$ & $6,4 \%$ & $7,5 \%$ \\
\hline Total & $100,0 \%$ & $100,0 \%$ & $100,0 \%$ & $100,0 \%$ \\
\hline
\end{tabular}

Source : Panel RICA-Source fiscale 1991-1997 (résultats non extrapolés).

La prise en compte des évolutions des exploitations en terme de risque financier entre 1991 et 1997 selon le statut en matière d'emploi (figure1) fait apparaitre pour les « foyers monoactifs en 1991 et 1997 » une amélioration sensible de leur position financière, dès lors que les effectifs présentant un risque faible, moyen et élevé régressent et que les effectifs des exploitations présentant un risque nul progressent au cours de la période considérée. Cette amélioration de la situation financière bénéficie également aux exploitations durablement concernées par la pluriactivité, puisque les effectifs des exploitations présentant un risque nul progressent et que les effectifs des exploitations présentant un risque financier élevé et faible se réduisent de manière drastique. Les exploitations dont le foyer est entré en pluriactivité entre 1991 et 1997, dont la situation financière initiale était plutôt dégradée, semblent avoir bénéficié d'une nette amélioration de leur position financière au cours de la période considérée. Les exploitations dont le foyer a cessé d'être pluriactif entre 1991 et 1997, qui avaient pourtant bénéficié d'une telle embellie dans un premier temps (Krebs, 2004), accusent finalement sur l'ensemble de la période étudiée une dégradation de leur position financière. La pluriactivité semble ainsi constituer un facteur propice à l'amélioration ou au rétablissement de la situation financière des exploitations. 
Figure 1. Evolution du risque financier entre 1991 et 1997 selon le statut en matière de pluriactivité

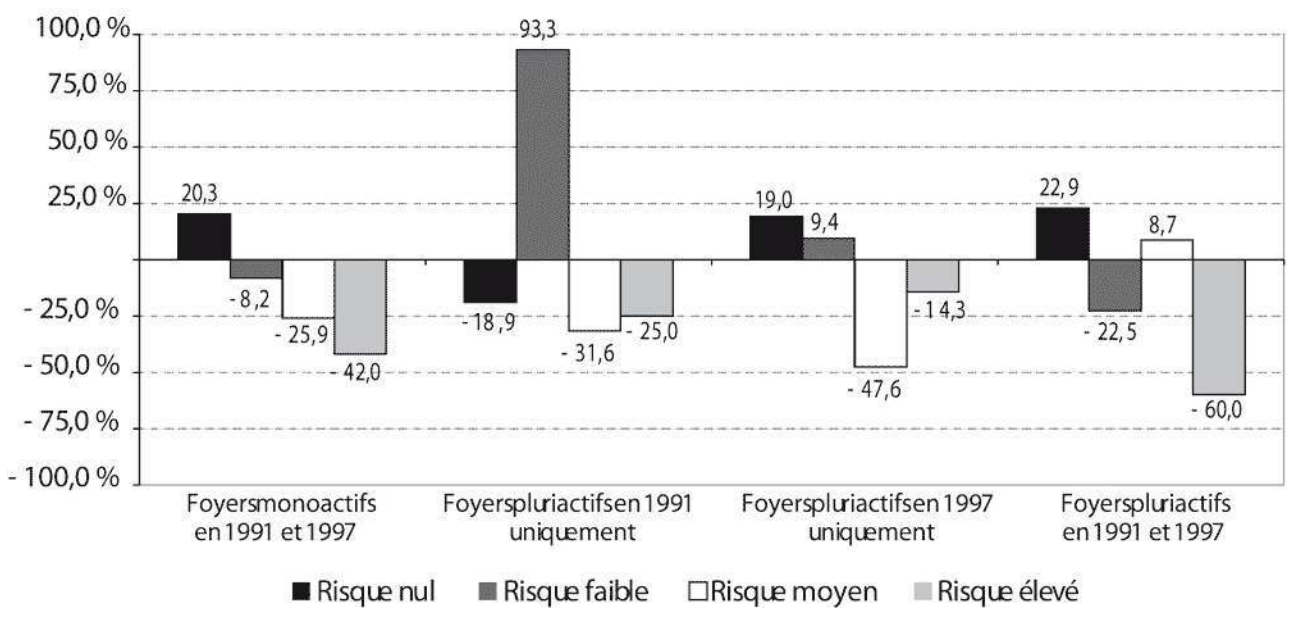

Source : Panel RICA-Source fiscale 1991-1997 (résultats non extrapolés).

Finalement, il apparaît que les préoccupations financières ne sont pas totalement absentes des décisions de travail des foyers d'agriculteurs. Des considérations financières peuvent justifier la fréquence plus élevée de la pluriactivité sur les exploitations positionnées en début de cycle de vie, lorsque l'exploitation doit faire face à d'importants besoins de financement liés à l'installation et à la modernisation de l'exploitation (tableau 1). Ces besoins importants en capitaux ne pouvant être couverts à partir des seules ressources financières internes dégagées par l'exploitation, il en résulte une généralisation du recours au financement bancaire. L'endettement élevé qui en résulte, qui peut s'avérer excessif pour certaines catégories d'exploitations (celles confrontées à des difficultés sectorielles par exemple) peut motiver leur entrée en pluriactivité, afin de percevoir un complément de ressources, nécessaire pour leur permettre de faire face à leurs obligations financières sans compromettre le niveau de vie familial. La décision d'entrée - ou de se maintenir - en pluriactivité peut également s'insérer dans la stratégie de croissance de l'exploitation, en particulier lorsque cette dernière est confrontée à l'existence d'imperfections des marchés de capitaux, dès lors que la perception de revenus d'activité extérieure est de nature à agir sur les conditions d'accès à la ressource financière, tant au plan du financement interne qu'au plan du financement externe. Les décisions de travail des foyers d'agriculteurs demeurent toutefois fortement conditionnées par certaines caractéristiques structurelles de l'exploitation (comme l'orientation technico-économique) qui peuvent rendre difficilement conciliable l'exercice simultané, par un même foyer, de deux activités professionnelles distinctes. C'est ce qui pourrait justifier la sortie de pluriactivité des "foyers pluriactifs en 1991 uniquement ", en dépit de la détérioration sévère de leur position financière. Cette dernière conclusion demeure toutefois à nuancer, du fait de la faiblesse des effectifs concernés par un tel changement de statut ${ }^{7}$. L'approche descriptive se doit désormais d'être complétée par une analyse économétrique des décisions de financement, en tenant compte des changements de statut des foyers d'agriculteurs en matière de pluriactivité intervenus entre 1991 et 1997. 


\section{Pluriactivité et financement interne de l'exploitation}

17 Il nous a semblé pertinent de mettre en premier lieu l'accent sur le financement interne de l'exploitation agricole. Le recours à des données d'origine comptable nous a conduit à proposer un cadre d'analyse original, consistant en l'estimation d'équations dynamiques de prélèvements privés. Cette approche, inspirée de la littérature économique relative aux comportements de consommation et d'épargne, vise à tester économétriquement la sensibilité des comportements de prélèvements à un ensemble de facteurs explicatifs, en tenant explicitement compte des changements de statut des foyers d'agriculteurs en matière d'emploi survenus entre 1991 et 1997. Les variables explicatives du montant des prélèvements courants - nets des apports familiaux - retenues sont le montant de ces prélèvements retardés d'une période, les résultats économiques courants et retardés de l'exploitation, la richesse nette en début de période, l'encours de dettes à court terme, ainsi que l'encours de dettes à long et moyen terme ${ }^{8}$. Les résultats obtenus font apparaître des comportements de prélèvements différenciés (tableau 5).

Tableau 5. Résultats de l'estimation de l'équation dynamique de prélèvements

\begin{tabular}{|c|c|c|c|c|c|}
\hline & Foyers & Foyers & Foyers & Foyers & Ensemble \\
\hline & monoactifs & pluriactifs & pluriactifs & pluriactifs & des \\
\hline & $\begin{array}{c}\text { en } 1991 \text { et } \\
1997\end{array}$ & $\begin{array}{c}\text { en } 1991 \\
\text { uniquement }\end{array}$ & $\begin{array}{c}\text { en } 1997 \\
\text { uniquement }\end{array}$ & $\begin{array}{c}\text { en } 1991 \text { et } \\
1997\end{array}$ & exploitations \\
\hline \multirow{2}{*}{$\begin{array}{l}\text { Prélèvements privés } \\
(\mathrm{t}-1)\end{array}$} & $-0,406^{* * *}$ & 0,071 & $-0,205^{* * *}$ & $-0,165$ & $-0,186^{*}$ \\
\hline & $(0,102)$ & $(0,050)$ & $(0,064)$ & $(0,128)$ & $(0,108)$ \\
\hline \multirow{2}{*}{$\begin{array}{l}\text { Capacité } \\
\text { d'autofinancement } \\
\text { (t) }\end{array}$} & $0,402^{* * *}$ & 0,154 & $-0,613^{* * *}$ & $0,499 * * *$ & $0,287^{* *}$ \\
\hline & $(0,111)$ & $(0,098)$ & $(0,170)$ & $(0,140)$ & $(0,134)$ \\
\hline \multirow{2}{*}{$\begin{array}{l}\text { Capacité } \\
\text { d'autofinancement } \\
(t-1)\end{array}$} & $0,325^{* * *}$ & $-0,064$ & $0,253^{* *}$ & $0,196^{*}$ & $0,250 * * *$ \\
\hline & $(0,078)$ & $(0,120)$ & $(0,101)$ & $(0,107)$ & $(0,085)$ \\
\hline \multirow{2}{*}{$\begin{array}{l}\text { Capacité } \\
\text { d'autofinancement } \\
(\mathrm{t}-2)\end{array}$} & $0,132 * * *$ & $0,445^{* * *}$ & 0,041 & 0,045 & $0,100 * *$ \\
\hline & $(0,034)$ & $(0,054)$ & $(0,056)$ & $(0,059)$ & $(0,040)$ \\
\hline Richesse nette (t) & 0,034 & $0,165^{* * *}$ & 0,064 & $0,250^{* *}$ & 0,030 \\
\hline
\end{tabular}




\begin{tabular}{|l|l|l|l|l|l|}
\hline & $(0,069)$ & $(0,055)$ & $(0,044)$ & $(0,126)$ & $(0,064)$ \\
\hline $\begin{array}{l}\text { Encours de dettes à } \\
\text { CT (t) }\end{array}$ & $\mathbf{0 , 6 0 5 * * *}$ & $\mathbf{0 , 3 4 7 ^ { * }}$ & $\mathbf{0 , 6 4 3} * * *$ & $-\mathbf{0 , 4 1 3 * * *}$ & 0,397 \\
\hline & $(0,210)$ & $(0,198)$ & $(0,103)$ & $(0,118)$ & $(0,242)$ \\
\hline $\begin{array}{l}\text { Encours de dettes à } \\
\text { LMT (t) }\end{array}$ & 0,092 & $-0,037$ & $-\mathbf{0 , 1 8 1 ^ { * * * }}$ & $-\mathbf{0 , 3 3 0 ^ { * * }}$ & 0,019 \\
\hline \hline Sargan & $(0,100)$ & $(0,089)$ & $(0,067)$ & $(0,154)$ & $(0,120)$ \\
\hline \hline -value & $\mathbf{1 3 , 1 7}$ & $\mathbf{2 6 , 6 7}$ & $\mathbf{1 4 , 6 3}$ & $\mathbf{2 0 , 3 4}$ & $\mathbf{1 1 , 4}$ \\
\hline m1 (p-value) & 0,01 & 0,14 & 0,80 & 0,44 & 0,93 \\
\hline \hline m2 (p-value) & 0,26 & 0,00 & 0,00 & 0,00 & 0,00 \\
\hline
\end{tabular}

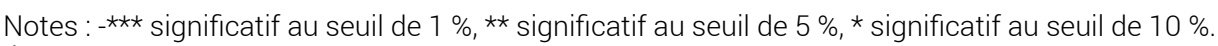
Écarts types robustes entre parenthèses.

-Les modèles ont été estimés en différences premières par la méthode des moments généralisés et incluent des indicatrices temporelles. La variable endogène retardée (en différences premières) a été instrumentée par ses propres retards (en niveaux), datées $t-3$ et $t-4$, les autres variables (en différences premières) étant instrumentées par leurs propres retards (en niveaux), datés t -2 . Les résultats présentés sont ceux de la deuxième étape.

Source : Panel RICA-Source fiscale 1991-1997

Les comportements de prélèvements des «foyers monoactifs en 1991 et 1997 », qui retirent l'essentiel de leurs revenus de l'exercice de l'activité agricole, semblent en effet particulièrement sensibles aux évolutions de leur environnement conjoncturel. Cette vulnérabilité les incite à tenir compte, lors du partage de leur revenu agricole, des résultats courants de l'exploitation, mais également des résultats passés de cette dernière, impliquant un effet persistant des résultats de l'exploitation sur leurs décisions de prélèvements. Ces dernières sont de plus influencées négativement par les comportements de prélèvements passés, traduisant le fait que les montants prélevés d'autant plus faibles que les sommes ponctionnées sur le revenu agricole ont été importantes par le passé. Les montants prélevés ne semblent en revanche pas influencés par la richesse nette - autrement dit par la capitalisation passée de l'exploitation-, ni par l'encours de dettes à long et moyen terme, ce qui signifie que le financement (passé) des investissements ne pèse pas sur le partage du revenu agricole.

19 En revanche, les montants des prélèvements sont influencés positivement par l'encours de dettes à court terme, ce qui implique que le financement du cycle de production par le biais de crédits à court terme est de nature à libérer des fonds, qui peuvent être alloués au financement des besoins familiaux.

La perception de revenus d'activité extérieure tend à rendre les comportements des " foyers pluriactifs en 1991 et 1997 » moins vulnérables aux évolutions conjoncturelles de leur environnement. La forte dimension temporelle, qui caractérisait les comportements des «foyers monoactifs en 1991 et 1997 », s'estompe dans le cas des foyers durablement 
pluriactifs. Leurs comportements de prélèvements ne subissent pas l'influence significative de leurs décisions passées de prélèvements et sont avant tout influencés par les résultats courants de l'exploitation - l'influence des résultats passés de l'exploitation étant fortement atténuée. Ces comportements sont désormais influencés positivement par la capitalisation passée de l'exploitation et subissent une influence très nettement négative des deux variables d'endettement. La sensibilité du partage du revenu agricole à l'encours de dettes à court terme et à l'encours de dettes à long et moyen terme traduit un vif arbitrage lors du partage du revenu agricole entre le financement des besoins familiaux et la volonté affichée par cette catégorie de foyers de privilégier le remboursement de leurs dettes contractées dans le cadre du financement de leur cycle de production et de leurs projets d'investissement.

Les résultats obtenus pour les foyers concernés par un changement de statut en matière d'emploi au cours de la période étudiée doivent être maniés avec plus de précaution, du fait de la relative faiblesse des effectifs concernés. Pour les «foyers pluriactifs en 1991 uniquement ", la dimension temporelle est ainsi quasiment absente, puisque leurs comportements de prélèvements ne subissent pas d'influence significative des prélèvements passés et que l'influence des résultats présents et passés de l'exploitation est extrêmement réduite. Les comportements de prélèvements sont par contre assez fortement liés à la capitalisation passée, et dans une bien moindre mesure (coefficient positif, mais faiblement significatif), à l'encours de dettes à court terme. Le coefficient associé à l'encours de dettes à long et moyen terme est négatif, mais non significatif, impliquant une absence de sensibilité des comportements de prélèvements aux décisions passées d'investissement. Cela peut contribuer à expliquer la dégradation de la position financière observée pour cette catégorie d'exploitation, dès lors que les montants prélevés sont définis presque indépendamment des résultats de l'exploitation et que cette catégorie de foyer ne semble pas privilégier outre mesure le remboursement des dettes contractées. Pour les "foyers pluriactifs en 1997 uniquement", la dimension temporelle redevient plus marquée, puisque les prélèvements passés exercent une influence négative et très significative sur les comportements de prélèvements courants. L'influence des résultats courants de l'exploitation est également significative, mais négative, ce qui peut paraître a priori surprenant, mais cet effet négatif est toutefois contrebalancé par l'influence positive des résultats passés de l'exploitation. La richesse nette est sans influence déterminante sur les montants prélevés, à la différence des variables d'endettement. L'encours de dettes à court terme agit positivement et significativement sur les montants prélevés, l'encours de dettes à long et moyen terme négativement et significativement. Ces comportements peuvent expliquer le rétablissement de la position financière de ces exploitations, initialement particulièrement dégradée.

\section{Pluriactivité et financement externe de l'exploitation}

Une seconde alternative possible en matière d'analyse des relations que sont susceptibles d'entretenir le statut du foyer d'agriculteurs en matière d'emploi et le mode de financement de l'exploitation consiste à mettre l'accent sur le financement externe, en tenant explicitement compte des difficultés rencontrées par les exploitations dans leur accès à la ressource financière externe. La méthodologie retenue, inspirée de celle mise en œuvre dans une étude récente réalisée conjointement par la Banque de France et la Deutsche Bundesbank sur le financement des firmes industrielles françaises et 
allemandes (Sauvé et Scheuer, 1999), consiste en l'estimation d'équations dynamiques d'endettement, liant le taux d'endettement courant au taux d'endettement retardé, à la croissance de l'exploitation, à l'importance des garanties présentées, à la rentabilité de l'exploitation, au service de la dette et au coût du financement (Krebs, 2002) ${ }^{9}$. La prise en compte des changements de statuts en matière de pluriactivité intervenus entre 1991 et 1997 peut être menée - comme précédemment - en opposant d'abord les deux catégories de foyers non concernées par un changement de statut en matière d'emploi entre 1991 et 1997 (tableau 6).

Tableau 6. Résultats de l'estimation économétrique de l'équation dynamique d'endettement

\begin{tabular}{|c|c|c|c|c|c|}
\hline & Foyers & Foyers & Foyers & Foyers & Ensemble \\
\hline & monoactifs & pluriactifs & pluriactifs & pluriactifs & des \\
\hline & $\begin{array}{c}\text { en } 1991 \text { et } \\
1997\end{array}$ & $\begin{array}{c}\text { en } 1991 \\
\text { uniquement }\end{array}$ & $\begin{array}{c}\text { en } 1997 \\
\text { uniquement }\end{array}$ & $\begin{array}{c}\text { en } 1991 \text { et } \\
1997\end{array}$ & exploitations \\
\hline \multirow[t]{2}{*}{$\begin{array}{l}\text { Taux } \\
\text { d'endettement }(t \\
-1)\end{array}$} & $0,962^{* * *}$ & $0,700^{* * *}$ & $0,756^{* * *}$ & $0,832^{* * *}$ & $0,961^{* * *}$ \\
\hline & $(0,061)$ & $(0,228)$ & $(0,132)$ & $(0,101)$ & $(0,051)$ \\
\hline \multirow[t]{2}{*}{ Croissance $(\mathrm{t})$} & $0,096^{* * *}$ & $0,061^{* * *}$ & $0,106^{* *}$ & $0,147^{* * *}$ & $0,103^{* * *}$ \\
\hline & $(0,018)$ & $(0,023)$ & $(0,048)$ & $(0,019)$ & $(0,014)$ \\
\hline \multirow[t]{2}{*}{ Garanties (t) } & 0,054 & $-0,042$ & $0,407^{* * *}$ & 0,100 & $0,083^{* *}$ \\
\hline & $(0,037)$ & $(0,137)$ & $(0,101)$ & $(0,064)$ & $(0,033)$ \\
\hline \multirow[t]{2}{*}{ Rentabilité (t) } & $-0,050^{* * *}$ & $-0,130^{* * *}$ & $-0,025$ & $-0,075^{* * *}$ & $-0,059^{* * *}$ \\
\hline & $(0,009)$ & $(0,026)$ & $(0,027)$ & $(0,017)$ & $(0,009)$ \\
\hline \multirow[t]{2}{*}{$\begin{array}{lll}\text { Service de } & \text { la } \\
\text { dette }(t) & & \end{array}$} & $-0,057^{* * *}$ & $-0,353^{* * *}$ & $-0,141^{* * *}$ & $-0,05$ & $-0,083^{* * *}$ \\
\hline & $(0,017)$ & $(0,091)$ & $(0,054)$ & $(0,042)$ & $(0,018)$ \\
\hline \multirow[t]{2}{*}{$\begin{array}{l}\text { Coût du } \\
\text { financement }(t)\end{array}$} & $-0,114^{* * *}$ & $-0,029$ & $-0,042$ & $-0,038$ & $-0,076^{* * *}$ \\
\hline & $(0,026)$ & $(0,019)$ & $(0,037)$ & $(0,030)$ & $(0,025)$ \\
\hline Sargan & 10,3 & 4,1 & 8,1 & 5,1 & 10,4 \\
\hline p-value & 0,11 & 0,67 & 0,23 & 0,53 & 0,11 \\
\hline m1 (p-value) & 0,00 & 0,01 & 0,00 & 0,00 & 0,00 \\
\hline
\end{tabular}




\begin{tabular}{|l|l|l|l|l|l|}
\hline $\mathrm{m} 2$ (p-value) & 0,80 & 0,33 & 0,43 & 0,21 & 0,06 \\
\hline
\end{tabular}

Notes : - ** significatif au seuil de $1 \%$, ** significatif au seuil de $5 \%$, * significatif au seuil de $10 \%$.

Écarts types robustes entre parenthèses.

-Tous les modèles ont été estimés en différences premières par la méthode des moments généralisés et incluent des indicatrices temporelles. La variable endogène retardée (en différences premières) a été instrumentée par ses propres retards (en niveaux), datés $t-3$ et $t-4$. Les résultats de la seconde étape sont reportés dans le tableau.

Source : Panel RICA-Source fiscale 1991-1997.

Les comportements d'endettement des «foyers monoactifs en 1991 et 1997 » subissent ainsi une influence positive et significative du taux d'endettement retardé (synonyme d'une très forte inertie des comportements d'endettement) et de la croissance de l'exploitation, tandis qu'ils sont influencés négativement et significativement par la rentabilité de l'exploitation, le service de la dette et le coût du financement. L'importance des garanties présentées ne semble par contre pas affecter significativement les décisions d'endettement des foyers durablement monoactifs.

Dans le cas des "foyers pluriactifs en 1991 et 1997 », l'inertie des comportements d'endettement demeure fortement significative, même si elle semble moins accentuée que celle observée pour leurs homologues durablement monoactifs, ce qui peut s'expliquer par le plus fort dynamisme des foyers d'agriculteurs pluriactifs. L'endettement est en effet particulièrement sensible à la croissance de l'exploitation, le recours à l'endettement étant d'autant plus intensif que l'exploitation doit faire face à d'importants besoins en capitaux, liés au financement de sa croissance. Le recours à l'endettement des foyers pluriactifs tend cependant à être freiné lorsque l'exploitation est caractérisée par une rentabilité élevée, donnant naissance à une hiérarchie du financement, l'exploitation durablement pluriactive préférant se financer en priorité directement à partir des résultats de l'exploitation avant d'envisager de contracter de nouvelles dettes financières. Cette catégorie d'exploitation ne semble pas confrontée outre mesure à des difficultés d'accès à la ressource financière externe, puisque les coefficients associés aux variables de garanties et de risque sont non significatifs, la perception de revenus d'activité extérieure par ces foyers étant de nature à leur permettre de supporter un endettement élevé sans risque excessif de défaillance. Enfin, à la différence de leurs homologues durablement monoactifs, l'endettement des foyers durablement pluriactifs n'affiche pas de sensibilité réelle au coût du financement.

Les comportements d'endettement des "foyers pluriactifs en 1991 uniquement " sont ainsi caractérisés par une inertie réduite, en dépit d'une faible sensibilité à la croissance de l'exploitation. Les choix financiers de ces foyers affichent par contre une sensibilité particulièrement importante à la rentabilité de l'exploitation, ce qui plaide en faveur d'une hiérarchie des sources de financement. Les décisions d'endettement ne subissent par contre pas d'influence significative des garanties présentées, mais sont par contre extrêmement sensibles au service de la dette, autrement dit au risque potentiel de défaillance de l'exploitation. Comme pour les «foyers pluriactifs en 1991 et 1997 », le coût du financement ne semble pas exercer d'influence significative sur le mode de financement. Dans le cas des "foyers pluriactifs en 1997 uniquement ", l'inertie des comportements d'endettement est également atténuée, comme d'ailleurs l'influence de la stratégie de croissance de l'exploitation. Les décisions financières de ces foyers ne semblent pas subir d'influence déterminante de la rentabilité, invalidant l'hypothèse de hiérarchie du 
financement pour ce sous-échantillon. Les exploitations concernées, tributaires du recours au crédit bancaire, semblent de plus confrontées à des difficultés d'accès à la ressource financière puisque l'influence du service de la dette est particulièrement nette et que l'endettement de cette catégorie d'exploitation est extrêmement sensible aux garanties présentées. A l'instar des autres catégories d'exploitations pluriactives, on ne note pas de sensibilité particulière au coût du financement.

\section{Conclusion} cette étude, qui sont pour l'essentiel inhérentes aux sources employées pour la construction de notre panel RICA-Source fiscale 1991-1997. La première limite concerne la population étudiée, puisque l'emploi des données comptables nous a conduit à écarter du champ de l'étude les exploitations non professionnelles relevant du champ de l'agriculture de complément, ainsi que les exploitations professionnelles de faible dimension. La seconde limite vient du fait que le champ couvert par le panel a encore été rétréci par l'exclusion des exploitations organisées sous forme sociétaire, ce qui est de nature à sous-estimer le phénomène de la pluriactivité et introduire un biais dans nos analyses empiriques. rare et incontournable de renouvellement des connaissances en matière de pluriactivité du fait de la fiabilité des données que permet la mise en relation de l'information comptable et de l'information fiscale. Il nous a permis, dans le présent article, de faire ressortir un certain nombre de spécificités des exploitations concernées par la pluriactivité, tant au regard du financement interne que du financement externe. La perception de revenus d'activité extérieure semble en effet permettre aux ménages concernés de réinvestir une part plus importante de son revenu agricole sur l'exploitation, du fait de la possibilité de financer directement les activités relevant de leur sphère privée à partir du revenus non agricole perçus, mais également du fait d'une moindre vulnérabilité aux évolutions de leur environnement. Les revenus d'activité extérieure semblent également agir sur la disponibilité de la ressource financière externe, en permettant notamment aux ménages pluriactifs de faire plus facilement face à un endettement important, sans exposer la banque à un risque excessif de défaillance.

Le panel RICA-Source fiscale ouvre enfin d'intéressantes perspectives de recherches futures. La voie de recherche la plus évidente concerne bien évidemment l'exploration sur données de panel des déterminants des décisions de participation à une activité extérieure, mais d'autres pistes, relatives à la contribution des revenus extérieurs à la gestion du risque de revenu ou encore à l'impact de la pluriactivité sur la croissance de l'exploitation, sont également envisageables. 


\section{BIBLIOGRAPHIE}

Arellano M. et Bond S. Some Tests of Specification for Panel Data: Monte Carlo Evidence and an Application to Employment Equations. Review of Economic Studies, 1991, vol. 58, p. 277-297.

Brangeon J.-L., Jégouzo G. et Roze B. Les revenus agricoles négatifs. Economie Rurale, 1999, n²24, p. 32-38.

Butault J.-P., Delame N., Krebs S., Lerouvillois p. La pluriactivité : un correctif aux inégalités du revenu agricole. Economie et Statistique, 1999, n³29-330, p. 165-180.

Colson F., Chatellier V., Blogowski A. Pour mieux comprendre les difficultés financières des agriculteurs. Agreste-Cahiers, 1995, n² 23, p. 3-8.

Lass D.-A., Findeis J.-L., Hallberg M.-C. Factors Affecting the Supply of Off-Farm Labor: A Review of Empirical Evidence In Hallberg M.-C., Findeis J.-L., Lass D.-A. (Eds) "Multiple Job-holding Among Farm Families", Iowa State University, 1991, p. 239-262.

Krebs S. L'accès au crédit des exploitations agricole. Synthèses, 2002, n 63, p. 119-134.

Krebs S. Activités non agricoles et financement de l'exploitation agricole. Thèse de Doctorat, Université Nancy 2, 2004.

Sauvé A., Scheuer M. (Eds). Mode de financement des entreprises allemandes et françaises. Rapport de recherche commun de la Deutsche Bundesbank et de la Banque de France, 1999.

Schmitt G. Farms, Farm Households, and Productivity of Resource Use in Agriculture.European Review of Agricultural Economics, 1989, vol. 16, p. 257-284.

\section{ANNEXES}

\section{Analyse économétrique}

Les travaux empiriques menés dans la présente étude visent à tester la sensibilité des décisions de financement des firmes agricoles à un certain nombre de facteurs explicatifs, en tenant explicitement compte de leur statut en matière d'emploi.

\section{Equation dynamique de prélèvements}

L'équation de prélèvements que nous nous proposons d'estimer est la suivante :

$$
\begin{aligned}
P_{i t}=\alpha_{1} P_{i, t-1}+\alpha_{2} C A F_{i t}+\alpha_{3} C A F_{i, t-1} & +\alpha_{4} C A F_{i, t-2} \\
& +\alpha_{5} N_{i t}+\alpha_{6} C T_{i t}+\alpha_{7} L M T_{i t}+\rho_{i}+\eta_{t}+\varepsilon_{i t}
\end{aligned}
$$

avec $i=1, \ldots, N$ et $t=1, \ldots, T$. 
La variable $P_{i t}$ désigne le montant des prélèvements privés courants (nets des apports familiaux et des charges sociales de l'exploitant) opérés par le ménage $i$ au cours de l'exercice comptable $t, C A F_{i t}$ désigne la capacité d'autofinancement courante (nette des charges sociales de l'exploitant), $N W_{i t}$ le patrimoine professionnel net de l'exploitation (approximé par l'actif total du bilan en début de période, diminué de l'endettement total en début de période), $C T_{i t}$ l'encours de dettes à court terme en début de période (dettes financières et non financières) et $L M T_{i t}$ l'encours de dettes à long et moyen terme en début de période. Le modèle fait également intervenir des effets fixes individuels $\rho_{i}$, destinés à prendre en compte des facteurs explicatifs non disponibles et/ou non observables spécifiques à chaque foyer d'agriculteur, ainsi que des effets fixes temporels $\mathrm{ht}$, afin de tenir compte de variables macro-économiques non disponibles et/ou non observables, non constantes au cours du temps et susceptibles d'affecter simultanément les comportements de tous les foyers d'agriculteurs représentés dans le panel RICASource fiscale. Enfin le modèle fait intervenir un terme d'erreur aléatoire, noté $\varepsilon_{i t}$.

\section{Equation dynamique d'endettement}

L'équation d'endettement à estimer est du type :

$E N D_{i t}=\alpha_{1} E N D_{i, t}-1+\alpha_{2} D Y N_{i t}+\alpha_{3} G A R_{i t}$

$+\alpha_{4} R E N_{i t}+\alpha_{5} S R_{i t}+\alpha_{6} \operatorname{COU}_{i t}+\rho_{i}+\eta_{t}+\varepsilon_{i t}$

avec $i=1, \ldots, N$ et $t=1, \ldots, t$.

La variable $E N D_{i t}$ désigne le taux d'endettement de l'exploitation $i$ à la fin de l'exercice comptable $t$ (obtenu en rapportant l'endettement total au total du passif du bilan), DYN la dynamique de croissance de l'exploitation (appréciée à l'aune du taux de croissance du total du bilan), GAR $R_{i t}$ les garanties présentées par l'exploitation (approximées en rapportant la valeur de l'actif immobilisé au total du bilan), $R E N_{i t}$ la rentabilité de l'exploitation (approximée en rapportant le revenu agricole brut au chiffre d'affaire), SER it le service de la dette (défini en rapportant l'annuité en capital à long et moyen terme, augmenté des frais financiers à court terme, au produit brut) et $\operatorname{COU}_{i t}$ le coût du financement de l'exploitation (apprécié à l'aune du taux d'intérêt apparent, défini en rapportant le montant des charges financières à long et moyen terme à l'encours de dettes à long et moyen terme). Comme précédemment, $\rho_{i}$ désigne l'effet fixe individuel, $\eta_{t}$ l'effet temporel et $\Sigma_{i t}$ un terme aléatoire. Notons encore que du fait de l'origine comptable des données, il n'a été possible de tenir compte lors de la construction de la variable « garanties » que des seules garanties physiques, la source statistique ne contenant aucune information relative aux autres types de garanties parfois exigées par les établissements bancaires (cautions, gages, etc.).

\section{Stratégie d'estimation}

L'estimation économétrique d'équations dynamiques sur données de panel soulève deux problèmes importants, liés à la présence parmi les régresseurs d'effets fixes individuels d'une part et de la variable endogène retardée d'autre part. Ces deux problèmes ne peuvent être solutionnés de manière satisfaisante à l'aide des outils de base de l'économétrie des données de panel et requièrent la mise en œuvre de techniques 
d'estimation plus sophistiquées. Le traitement des effets fixes individuels s'opère traditionnellement en procédant à l'estimation d'un modèle à effet fixe ou à l'estimation du modèle en ayant au préalable transformé les diverses variables en écarts aux moyennes individuelles (transformation Within). Cette dernière solution, qui présente l'avantage de faire disparaître l'effet fixe individuel de la relation à estimer, est toutefois de nature à entraîner une contamination de la perturbation $\Sigma_{i t}$ par les perturbations des autres périodes, présentes dans la perturbation individuelle moyenne $\varepsilon_{i^{*}}$. Ce phénomène survient typiquement lorsque les erreurs de mesure sont corrélées au cours du temps ou, comme cela est généralement le cas lors de l'estimation de modèles dynamiques sur données de panel, lorsque des variables explicatives retardées sont employées comme instruments (Sauvé et Scheuer, 1999). C'est ce qui motive finalement le recours à une troisième méthode, consistant en l'estimation des équations dynamiques de prélèvements et d'endettement en différences premières. L'estimation du modèle en différences premières est également motivée par la nécessité d'apporter une réponse satisfaisante au problème soulevé par la présence de la variable endogène retardée parmi les régresseurs, qui pose avec une certain acuité la question de la simultanéité de cette variable avec l'erreur résiduelle. Afin de surmonter ces difficultés, ainsi que d'autres difficultés potentielles liées à des erreurs de mesure des régresseurs, à des problèmes d'hétéroscédasticité ou à la corrélation temporelle des résidus, la méthode des variables instrumentales est fréquemment appliquée au modèle dynamique, préalablement réécrit en différences premières. Arellano et Bond (1991) ont ainsi proposé une méthode d'estimation en deux étapes, reposant sur l'emploi de la Méthode des Moments Généralisés, qui permet à partir des conditions d'orthogonalité sur la matrice de variance-covariance, de traiter les problèmes de biais de simultanéité, d'hétéroscédasticité, de corrélation des erreurs dans le temps ou encore d'erreurs de mesure.

La principale difficulté rencontrée avec ce type d'approche réside toutefois dans le choix des instruments. Ce délicat problème nous a conduit à procéder à l'estimation d'un nombre importants de variantes des équations dynamiques de prélèvements et d'endettement. Seuls les modèles les plus pertinents sont présentés dans les tableaux. Les tests statistiques qui accompagnent les résultats (test de Sargan, statistique $m_{2}$, permettant d'apprécier la validité des estimations par la Méthode des Moments Généralisés.

Notons enfin que l'estimation en différences premières, la présence de variables retardées et l'instrumentation de ces dernières induisent le raccourcissement de la période d'estimation à quatre années (1994-1997), contre sept (1991-1997) pour la période d'estimation.

\section{NOTES}

1. OCDE. Impact de la réforme de la politique agricole commune sur l'emploi dans l'agriculture. Comité de l'agriculture, direction de l'alimentation, de l'agriculture et des pêcheries, 2001, document de travail, $47 \mathrm{p}$.

2. Cet article constitue une synthèse des principaux résultats empiriques obtenus dans le cadre d'une thèse de doctorat, cofinancée par l'Institut national de la recherche agronomique (INRA) et la Caisse nationale de crédit agricole (CNCA) et soutenue le 31 mars 2004 à l'Université Nancy 2.

3. Les exploitations organisées sous forme sociétaire (EARL, GAEC et autres) n'ont pu être prises en compte lors de la construction du panel, du fait de leur exclusion lors de l'enquête de 1991. 
4. Ces résultats divergents sont probablement pour partie imputables à l'exclusion des formes sociétaires et au cylindrage du panel, qui conduit à la non prise en compte des créations, reprises et disparitions d'exploitations. Or, la pluriactivité tend à être plus répandue sur les exploitations positionnées en début de cycle de vie et/ou organisées sous forme sociétaire.

5. Cette affirmation est toutefois à nuancer dans la mesure où les données disponibles ne permettent pas de disposer annuellement d'informations concernant les changements de statut en matière d'emploi entre 1991 et 1997. Les mouvements d'entrée et de sortie de pluriactivité peuvent ainsi être liés à une variation brutale du revenu d'activité extérieure, sans que celle-ci ne soit nécessairement imputable à un changement significatif de l'allocation du temps du ménage.

6. Les exploitations ont été classées en fonction de leur degré de risque financier en employant la méthodologie proposée par Colson, Chatellier et Blogowski (1995). Cette typologie associe trois indicateurs de la solvabilité de l'exploitation (le ratio service de la dette/produit brut, le ratio endettement total/actif du bilan et le ratio dettes à court terme/actif circulant). Pour chaque ratio, deux seuils sont définis : un seuil d'alerte, au delà duquel les équilibres financiers de l'exploitation sont fragilisés, et un seuil critique, au delà duquel la situation de l'exploitation peut être considérée comme particulièrement dégradée. Chacune des exploitations du panel a ainsi été rattachée à un groupe de risque selon sa position par rapport aux seuils retenus pour ces indicateurs financiers.

7. Ce mouvement de sortie de pluriactivité pourrait également renvoyer plus simplement à d'autres facteurs externes, d'ordre économique ou familial (perte d'emploi, maladie, etc.), de nature à entraîner une réduction sensible du montant du revenu d'activité non agricole déclaré au fisc.

8. Le lecteur intéressé par la définition des variables et la présentation de la stratégie d'estimation est invité à se reporter à l'encadré méthodologique en annexe du présent article.

9. Le lecteur intéressé par la définition des variables et la présentation de la stratégie d'estimation est invité à se reporter à l'encadré méthodologique en annexe du présent article.

\section{RÉSUMÉS}

Cet article se propose de mettre en relation les décisions de pluriactivité des familles d'agriculteurs et les choix qu'ils opèrent en matière de financement de leurs exploitations. Les résultats obtenus à partir d'un panel d'exploitations individuelles françaises font ressortir d'importantes spécificités des exploitations pluriactives, tant en matière d'autofinancement que d'endettement.

The aim of this article is to study the possible interrelationships between the off-farm work decisions of the farm household and the farm financial management decisions on their farm business. The results obtained using French panel data allow us to conclude to the specificity of the financing behaviour of pluriactive farms in regard to both internal and external finance. 
INDEX

Mots-clés : autofinancement, données de panel - RICA, endettement, pluriactivité

Keywords : external finance, internal finance, off-farm work, panel data - fadn

Code JEL Q12 - Micro Analysis of Farm Firms; Farm Households; and Farm Input Markets, Q14 Agricultural Finance

\section{AUTEUR}

\section{STÉPHANE KREBS}

Unité mixte de recherche ENGREF-INRA, Laboratoire d'économie forestière, Nancy 